\title{
RAPID DESIGN OF TOOL-WEAR CONDITION MONITORING SYSTEMS FOR TURNING PROCESSES USING NOVELTY DETECTION
}

\author{
A. Al-Habaibeh , A. Al-Azmi and J. Redgate \\ Advanced Design and Manufacturing Engineering Centre \\ School of the Built Environment \\ Nottingham Trent University \\ Burton Street, Nottingham, NG1 4BU,UK
}

\begin{abstract}
Condition monitoring systems of manufacturing processes have been recognised in recent years as one of the key technologies that provide the competitive advantage in many manufacturing environments. It is capable of providing essential means to reduce cost, increase productivity, improve quality and prevent damage to the machine or work-piece. Turning operations are considered one of the most common manufacturing processes in industry. It is used to manufacture different round objects such as shafts, spindles and pins. Despite recent development and intensive engineering research, the development of tool wear monitoring systems in turning is still on-going challenge. In this paper, force signals are used for monitoring tool wear in a feature fusion model. A novel approach for the design of condition monitoring systems for turning operations using novelty detection algorithm is presented. The results prove that the developed system can be used for rapid design of condition monitoring systems for turning operations to predict tool-wear.
\end{abstract}

Keywords: Condition Monitoring, novelty detection, turning, tool wear, sensor fusion.

\section{INTRODUCTION}

The global manufacturing competition in recent years has attracted the manufacturer's attention to the application of condition monitoring of machine tools and processes as a method of improving quality of products, eliminating inspection, and enhancing manufacturing productivity. Effective condition monitoring system depends mainly on the ability of the system to identify any faults and respond, in real time, with a suitable action. A condition monitoring system, as shown in Figure 1 consists of sensors, signal processing stages, and artificial intelligence (decision making) stages to interpret the information obtained by the sensors and to decide on the essential corrective action (Martin, 1994).

The capability of condition monitoring systems relies on two basic elements: first, the type of sensors used and second, the associated signal processing methods used to extract the necessary important information (sensory characteristic feature, SCF) from machining signals. The first element could involve expensive hardware which influences the cost of the system, while the second element affects the efficiency and the response of the system. The main aspect is to design a condition monitoring system with high efficiency, short development time, and with a reduced number of sensors (Al-Habaibeh and Gindy, 2001, Al-Habaibeh and Parkin, 2003).

Machining processes are extremely complicated systems when compared with other production systems. Designers of condition monitoring systems of machining processes are normally expected to fully understand the characteristic of the process in order to be able to design the correct monitoring system. This approach would require from the designer an extended time and experimental work to be able to understand and model the machining mechanisms involved. Such approach could be useful for optimising machine performance as well as the quality of the machined parts. Nevertheless, modelling a manufacturing processes and studying its dynamics might not be necessary for developing an industrial 
condition monitoring system for existing manufacturing processes. Therefore, experimental approach will be used in this paper to design a condition monitoring system for tool wear without the need to fully understand the cutting mechanism of this particular process.

Turning process is one of the most common machining processes in industry which is performed on a lathe machine. Turning process used to machine many types of products and round-shaped features. There are many types of faults associated with turning processes including surface roughness, catastrophic cutter breakage, gradual tool wear and collision. Many researchers have investigated tool wear in turning processes, see for example (Barschdorff et al., 1993, Sick, 2002, Choudhury and Kishore, 2000, Chung et al., 1993, Scheffer and Heyns, 2004, Li, 2002). However, different research methods have shown different results based on the difference in material, cutter type, machining parameters, etc.

Several condition monitoring strategies have been proposed and evaluated during the last 30 years, see for example (Dimla et al., 1997, Tlusty and Andrews, 1983, Tansel and McLaughlin, 1993, Cakir and Isik, 2005, Ko and Cho, 1994). Nevertheless, until now there is a limited attempt for the development of generic and universal approach for condition monitoring design and evaluation. The condition monitoring research in general is based on evaluation of process monitoring methods and pattern recognition techniques to identify a specific condition of a process or to identify abnormalities from normal conditions. Different attempts have been found in literature to aid designing of monitoring systems (Sokolowski and Kosomol, 1996, Sokolowski et al., 1997). The mentioned references describe a methodology of designing a monitoring system by choosing the best configuration of sensors and signal processing methods using Scatter Matrix Method and feed forward back propagation neural networks. (Azouzi and Guillot, 1997) present a systematic method for sensor selection and fusion in turning process using neural networks. Orthogonal Arrays where used to design the experiment, and the percent contribution of each machining parameter on the level of signals is evaluated. The Authors (Al-Habaibeh and Gindy, 2000, Al-Habaibeh and Gindy, 2001, Al-Habaibeh et al., 2002) have presented several research results in using a new approach of automating the design methodology of condition monitoring systems, called ASPS: Automated Signal and Signal Processing Selection method, using features extraction of sensory signals to select the most appropriate sensor and its associated signal processing methods in order to reduce cost and development time. For each sensory signal obtained, an attempt is made to extract sensitive "sensory characteristic features, SCF" that can be related to cutter conditions using wide range of signal analysis and simplification techniques. An association matrix, (ASM), is used to establish the dependence (i.e. sensitivity) of the signal's sensory characteristic features on process variables. For more details, see (Al-Habaibeh et al., 2002, Al-Habaibeh and Gindy, 2001, Al-Habaibeh and Gindy, 2000).

This paper investigates the capability of the ASPS approach in turning using tool wear as the monitored fault. Turning process has been performed from fresh cutter to totally damaged cutters. Novelty detection algorithm is then used to classify the signal and to design the complete monitoring system.

\section{TOOL WEAR MONITORING}

Research in the last two decades shows that measuring cutting forces is one of the most commonly used methods for monitoring systems since these parameters are relatively easy to measure using dynamometer mounted on the tool holder, and is more sensitive to tool condition, see for example (Dornfeld, 1990, Martin, 1994). However, the there is no clear systematic methodology for the design process. This paper examines the ASPS approach for the design of condition monitoring systems using force signals.

Force is found suitable to measure tool breakage; the three cutting forces, the tangential force, the feed force and the normal force, when the broken tool nose is jammed between the workpiece and the tool, the forces, are found that they increase suddenly and dropped to zero as the broken part of the tool insert is 
released because of the gap between the tool and the workpiece (Tlusty and Andrews, 1983). By comparison, (Dornfeld and Lan, 1984) finds that the tangential force decreases as the insert broke while the feed force might decrease or increase depending on the cutting edge. References (Dimla Sr., 2000, Dimla Sr. and Lister, 2000b, Dimla Sr. and Lister, 2000a) use a force dynamometer to acquire cutting force data in the time and frequency domain. The references propose that when the tool wear gets to catastrophic failure, this will appear in the amplitude of the frequency associated with the changes in the dynamic force. Reference (Lee et al., 1992) recognises the relationship between the wear and the tangential force, the amplitude of the dynamic force increases with tool wear and decreases just before tool failure. Reference (Lee et al., 1996) proposes and implements a model for tool wear prediction using cutting force signals. Turning tests are performed on a lathe and the required data collected and resulting wear measured. A number of tests are initially performed at a variety of cutting conditions. From these tests, the force components least sensitive to process parameter change but most sensitive to tool wear are identified (Das et al., 1996). Obtained results indicate close correlation between actual data and the estimated values. With varying cutting speed and depth of cut, an error estimate of 6-9.5\% is reported for constant feed-rate, chip breaker and workpiece data. Therefore, this error is high for tool wear prediction. Reference (Elanyar and Shin, 1995) proposes a combined method of cutting tool flank and crater wear estimation. In the implementation stage of their method, data from experiments performed on a vertical lathe are used. The experiments involved recording the pre-processed (low pass filtering) components of the cutting forces from two sets of tests conducted. For the first set of tests, they use coated carbide inserts and carry out the tests at low cutting speeds. The second set of tests uses ceramic inserts and is carried out at higher speeds. The same workpiece material, feed-rate and depth of cut are utilised in both test sets. Force-wear relationships partly based on the same principle as reported in (Purushothaman and Srinivasa, 1994, Elanayar et al., 1990) are employed to select the input coefficients for training the network. Results showed it to be capable of close flank wear estimation but poor crater wear estimation. Overall, the proposed method demonstrats an ability to estimate tool wear closely, over a wide range of cutting conditions but lacked vital accuracy. The inaccuracies could be attributed to poor input coefficient estimation; they relate the cutting forces to tool wear with liner equations. It is therefore misleading to contemplate combining the two data types and expect unanimity.

References (Zhou et al., 1995, Rahman et al., 1995, Hong et al., 1996) performe cutting tests from which they record the dynamic cutting forces signature. A dimensional space features selection process is carried out and three different implementations are implemented. In (Zhou et al., 1995) researchers use a wavelet technique to extract such features from the convoluted force signal. Results obtained showed inaccuracies due to changed chip shape as a result of tool state change. The main reason for this, as claimed, is that in any cutting process more than two classes of cutting states actually existed, and a network with just one output can only recognise two of them. Thus, the final classification results would not be entirely correct.

References (Purushothaman and Srinivasa, 1994, Elanayar et al., 1990, Elanyar and Shin, 1995, Yao and Fang, 1993) also utilise the cutting force signals. The net output gave an indication of the product surface finish established earlier as representative of the tool state. Effectively, reference (Elanayar et al., 1990) utilised this experiment to relate tool wear, surface finish, and cutting forces and reported a high but unquantified achievement rate. Reference (Purushothaman and Srinivasa, 1994) approach and methodology is similar to that of (Rangwala and Dornfield, 1987), but differs in terms of sensor signal inputs, data processing. Reference (Purushothaman and Srinivasa, 1994) argues that there is no need to use dimensionally selected features, instead, they use the amplified components of the cutting force signal as sampled, together with the three cutting parameters (Rangwala and Dornfield, 1987, Dornfeld, 1990).

Reference (Tanner and Loh, 1994) utilises the cutting forces as their sensor signal inputs with an additional component depiction the occurrence of tool breakage to monitor the cutting process. The reference incorporated the cutting condition parameters (cutting speed, radial and axial depth of cut) to their chosen sensor signals and workpiece material, 
Reference (Kakade and et, 1995) integrates cutting forces and AE signals to investigate the influence of flank wear on the individual signals. Machining test cuts are performed from which the cutting forces and the AE signals are recorded. They concluded that by combining different source signals for tool wear classification success rate significant improved the performance of the system.

Reference (Masory, 1991) carries out single point cutting test experiments. It measures and records the tool tip temperature, the true root mean squared value (RMS) of the AE signal, and the three components of the cutting forces. The reference reportedly achieved very precise wear evaluation but no quantification of the results is provided.

Reference (Monostori, 1993) uses the vibration and cutting forces from both turning and milling tests to perform tool state classification, continuous estimation of tool wear and the inverse modelling of the cutting process. Reference (Monostori, 1993) experiments involve carrying out milling and turning test cuts utilising both fresh and worn inserts, recording the cutting forces, and vibration components from each cut. Using the measured signals, statistical and spectral features were computed, followed by performance of sequential forward search or feature selection process using the same selection criteria as (Dornfeld, 1990, Rangwala and Dornfield, 1987, Monostori, 1993) by using the force data to perform classification tests.

From the above discussion, it can be clearly concluded that force signals could be utilised for condition monitoring applications. However, there is a need for a systematic design methodology in order to utilise the right features for the design of a suitable condition monitoring system.

\section{NOVELTY DETECTION}

Novelty detection is used in this work as a self-learning approach to characterise the "fresh" or normal state of the cutter. Novelty detection (Al-Habaibeh et al., 2005, Zorriassatine, 2000) is a classification technique that recognises a presented data to be novel (i.e. new) or non-novel (i.e. normal). The training data for the novelty detection algorithm consists of only the normal class which is often much easier to obtain. Since a degree of overlap is normally expected between different classes, classification problems have a probabilistic nature(Bishop, 1995). Novelty detection involves estimating the probability-densityfunction $(P D F)$ of a normal class from the training data and then estimating the probability that a new set of data belongs to the same class. The classification decision in novelty detection is based on Bayes' theorem as shown in equation (1).

$P\left(C_{i} \mid x\right)=\frac{p\left(x \mid C_{i}\right) \cdot P\left(C_{i}\right)}{p(x)}$

Where

$P\left(C_{i} \mid x\right)$ : The Posterior Probability, the probability that a given vector, $x$, belongs to class $C_{i}$.

$P\left(C_{i}\right)$ : The Prior Probability, the probability that a future input, $x$, belongs to a class,$C_{i}$, based on the ratio of training examples that belong to the same class.

$p\left(x \mid C_{i}\right): \quad$ The Class-Conditional Probability Density, the probability of obtaining an input vector from a given class based on estimating the PDF of a class.

$p(x)$ : $\quad$ Unconditional Probability Density, probability density of $x$ regardless of the which class it belongs to. 
The Unconditional Probability Density should also satisfy the following equation:

$$
p(x)=\sum_{i=1}^{k} p\left(x \mid C_{i}\right) \cdot P\left(C_{i}\right)
$$

\section{Where}

$0 \leq P\left(C_{i}\right) \leq 1$ and

$\sum_{i=1}^{k} P\left(C_{i}\right)=1$

The accuracy of novelty detection classification is dependent on the accuracy of the modelled density functions (Zorriassatine, 2000). Three main methods are normally used to model the PDF: Parametric methods (Fukunaga, 1990), Non-Parametric methods (Parzen, 1962) and Semi-Parametric method (Specht, 1990). The parametric methods assume sufficient statistical information about the training data set which is not normally available. In non-parametric methods no assumptions are made regarding the underlying density functions and they depend on the training data to find the probability density function for a new input. (Bishop, 1995) classifies such methods as being Kernel based techniques and K-Nearest Neighbour technique. The K-nearest neighbour method depends on the probability that K number of data points of a vector fall within a specific volume. The Kernel-based technique calculates the volume by defining width parameters for a number of known probability distribution functions (Kernels) to provide a general model for the training set. However, non-parametric methods require long computations for every input vector. Semi-Parametric density estimation is used in this paper for novelty detection because it combines the advantage of both Parametric and Non-Parametric techniques and does not require extensive computational effort. Semi parametric methods use fewer numbers of Kernels. A Gaussian Mixture Model (GMM) is used in this paper to estimate the PDF. Unlike non-parametric methods the training data are used only during the process of construction of the density model and are not needed for calculation of the PDF for new vectors.

The probability density estimation of GMM is obtained by Bayes' theorem, similar to equation 2, as follows (Bishop, 1995):

$$
p(x)=\sum_{j=1}^{M} p(x \mid j) \cdot p(j)
$$

\section{Where}

$0 \leq p(j) \leq 1$

$\mathrm{M}$ is the number of components in the mixture model $p(j)$ is the Prior probability of selecting the jth kernel function $p(x \mid j)$ is the conditional density of $x$ on the jth kernel.

For a Gaussian Mixture Model, the following equation is derived from equation (3) (Bishop, 1995):

$$
p(x)=\sum_{j=1}^{M} \phi_{j}(x) \cdot \alpha_{j}
$$


Where

$\phi_{j}$ is the response of the jth Gaussian component

$\alpha_{j}$ is the mixing coefficient (priors) of $\phi_{j}$

When the probability distribution function is calculated. A threshold value can be used to define the boundaries between a novel vector and a normal data set (Zorriassatine, 2000). Figure 2 explains the methodology through which the novelty detection is used in this work to detect faulty conditions. Novelty detection software NETLAB (Nabney and Bishop, 2000) is incorporated with Matlab programs as a decision making algorithm for the diagnostic and prognostic of tool wear. More details regarding the novelty detection can be found in (Nabney and Bishop, 2000, Bishop, 1995).

\section{THE EXPERIMENTAL WORK}

The experimental work is performed on a lathe machine tool, as shown in Figure 3. It involves a turning process of mild steel parts using Titanium Carbide inserts. The chosen process parameters monitored are the cutting forces. The force signals are monitored using 3-component Dynamometer (Kistler 9257A). The force dynamometer is connected to a 3-channel charge Amplifier (Kistler 5001). The signals are monitored using a National Instrument NI PCI-6070E and a specially designed software using NI LabWindows/CVI. The sampling rate is $1 \mathrm{~K}$ Sample/s and turning process is performed at $2 \mathrm{~mm}$ depth of cut, $1000 \mathrm{RPM}$ spindle speed and $0.05 \mathrm{~mm} /$ revolution feed-rate. The steel work piece had a diameter of $20 \mathrm{~mm}$. Figure 4 presents the complete diagram of the experimental setup. Only Fx and Fz were monitored in this experimental work since Fy in this case was not significant.

\section{RESULTS AND DISCUSSION}

The experimental work for gradual tool wear is performed for 8 independent tests. Every test started with a fresh tool (insert) and finished with completely worn tool. An example of the raw signals for the first tool is shown in Figure 5. One test is used to investigate the process characteristic, select the sensitive SCFs and choose the sensitive sensors and signal processing methods. The SCFs of all the 8 tests are then fed into novelty detection algorithm to investigate the capability of the complete monitoring system.

Since turning produces complex machining signals, it might be difficult to predict the most sensitive signals and signal processing methods to cutter wear directly from the machining signals. Therefore, signal processing and analysis is needed to extract the important information in the signals. Normally, the analysis is performed manually by using different signal processing technique to evaluate the sensitive features to the fault under investigation. In order to extract the sensory characteristic features (SCFs), the 2 force signals are processed using several time and frequency domain signal processing methods to extract 16 SCFs form every sensory signal. The SCFs should be real numbers in order to be used in this approach (Al-Habaibeh and Gindy, 2001). The signal processing methods used in the time domain are: the average $(\mu)$; standard deviations $(s t d)$; power (Etter, 1993); kurtosis value $(K)$ (Wang and Dornfeld, 1992); and skew value (Wang and Dornfeld, 1992). In frequency domain two methods are used: the average values of different frequency ranges of fast Fourier transformation (FFT) using $100 \mathrm{~Hz}$ region for each feature.

The 16 signal processing methods are used to process the 2 sensory signals establishing an Association Matrix ASM of $(2 \times 16)$ which allows the investigation of 32 sensory characteristic features (SCFs) for the design of the monitoring system. The SCFs are arranged according to their sensitivities to cutter wear 
based on the absolute slope of the linear regression method. The SCFs are visually inspected and it has been found that SCFs with high absolute slope show higher sensitivity to the fault Figure 6 shows examples of high and low-sensitivity features to cutter wear. As it can be noticed from the figures, the absolute value of the slope of the least squares straight line fitting presents a good indication of how sensitive a sensory feature is to tool wear. Since the axes of both graphs are similar (normalised), the slope angle can be calculated from the line slope using the following equation:

$\theta^{\circ}=(180 / \pi) \cdot \tan ^{-1}($ slope $)$

From Figure 6 it could be concluded that the maximum value of $\mathrm{Fz}$ for each sample is much more sensitive to tool wear that The FFT value between 500 and $600 \mathrm{~Hz}$ for the same force signal. The other seven tests have shown consistent results. The SCFs with relatively high absolute slope show high sensitivity (i.e. relative change in the value) compared with SCFs with lower absolute slope values). By selecting the most sensitive group of SCFs, the monitoring system could be designed to investigate tool wear of the 8 tests.

NETLAB software is used (Nabney and Bishop, 2000) for the implementation of the novelty detection. The response of the Gaussian kernels $\phi_{j}$ (see Figure 2 and equation 4) is defined by a covariance matrix ( a spherical matrix in this case) and a centre (i.e. the centroid of the input clusters). A single variance parameter for each Gaussian component is calculated using 6 centres in the mixture which has been found to be a suitable structure that gives relatively quick learning process and consistent results.

Following the training of the novelty detection on normal samples using 40 machining samples from the first test out of 100 samples, the complete captured of normal and faulty features are tested. Figure 7 presents the results of using the novelty detection for testing the sensory characteristic features. By selecting a suitable threshold value the success of the novelty detection algorithms is found to be $100 \%$. Moreover, the threshold value could be selected for efficient tool wear prediction before the actual tool wear occurs.

\section{CONCLUSIONS}

This paper has presented the application of a self learning approach, ASPS, (Al-Habaibeh and Gindy, 2001) for the design of tool wear monitoring in turning. The applied approach helps in designing a condition monitoring system from experience using a simple automated algorithm to determine the sensory characteristic features (SCFs) which are most sensitive to process tool wear. The monitoring system has been tested using 8 deferent tools. Examples of low and high sensitivity SCFs have been presented. Novelty detection has been implemented as an artificial intelligence and decision making stage. The results found prove that the combination of sensitive sensory characteristic features and novelty detection algorithm have been successful for tool wear detection and predication. 


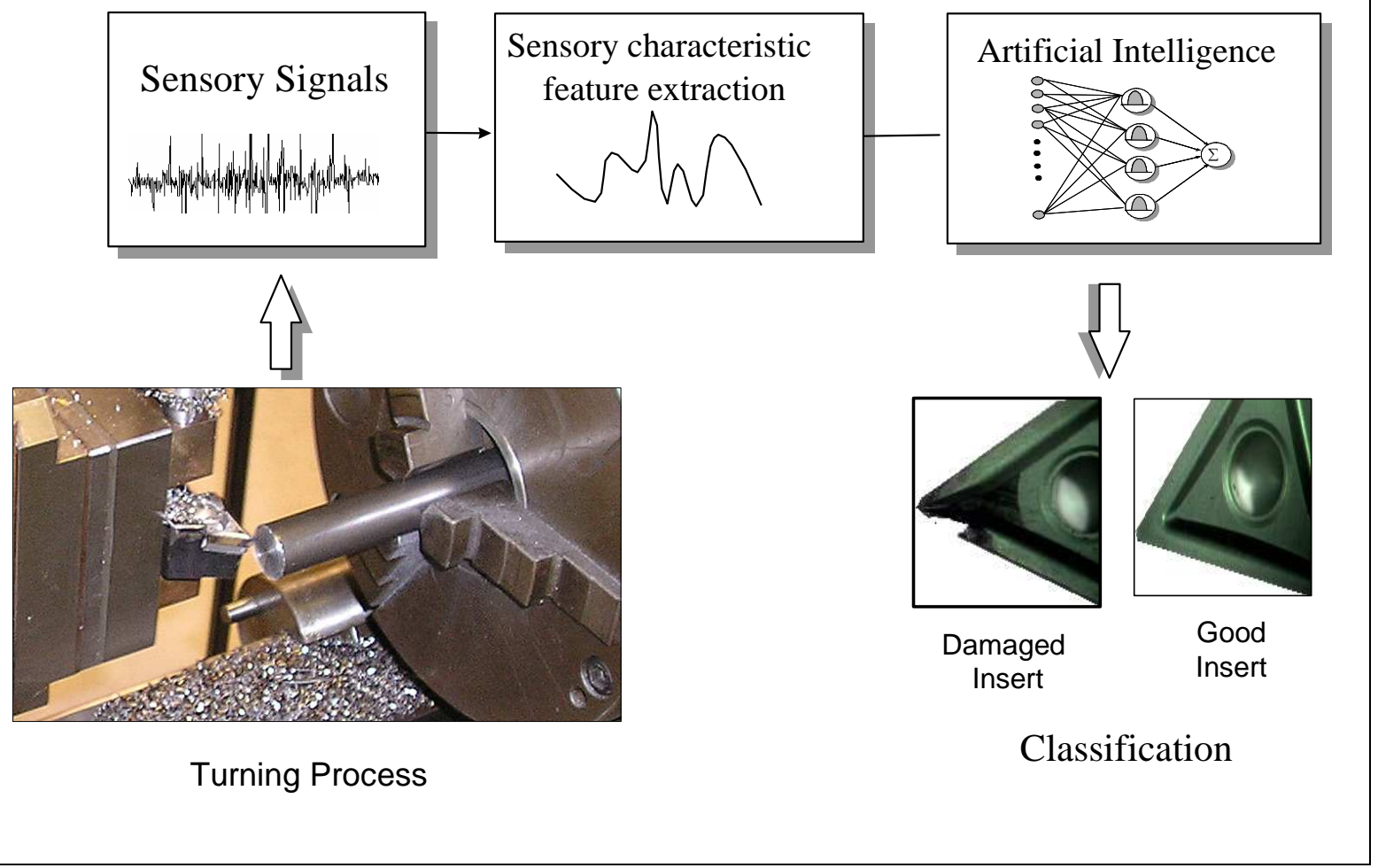

Figure 1: The general structure of a condition monitoring system.

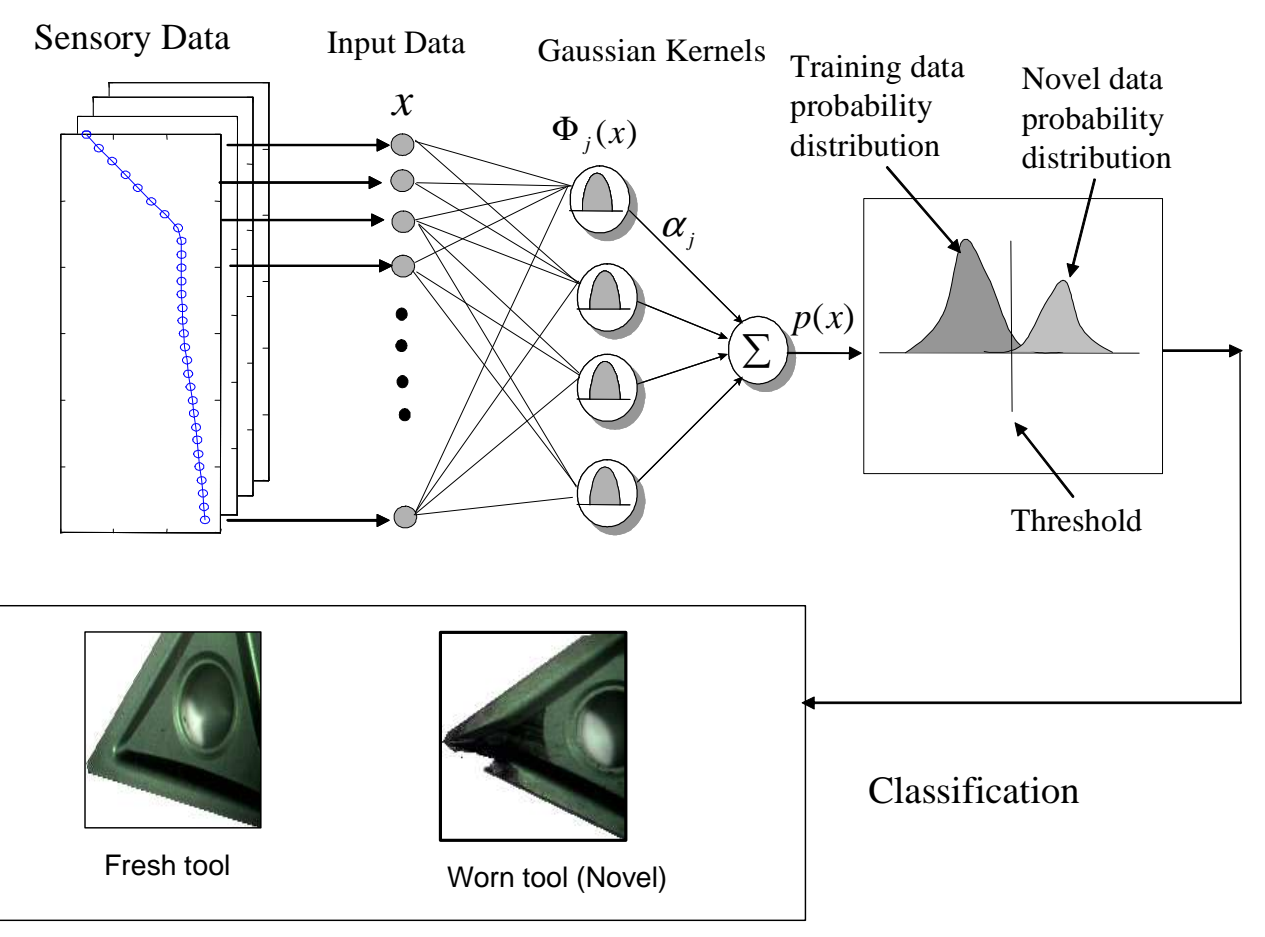

Figure 2: The application of novelty detection for monitoring gradual tool wear in turning. 


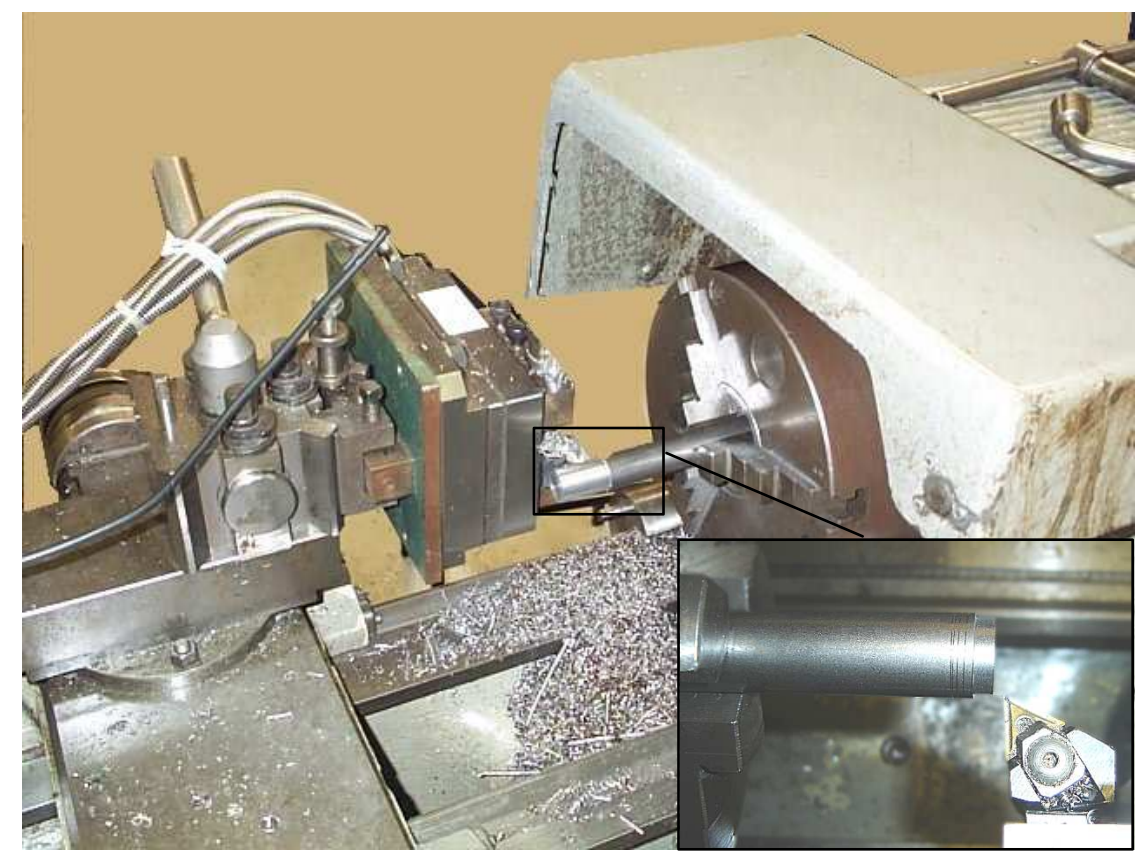

Figure 3: A schematic diagram of the experimental set-up using a force dynamometer.

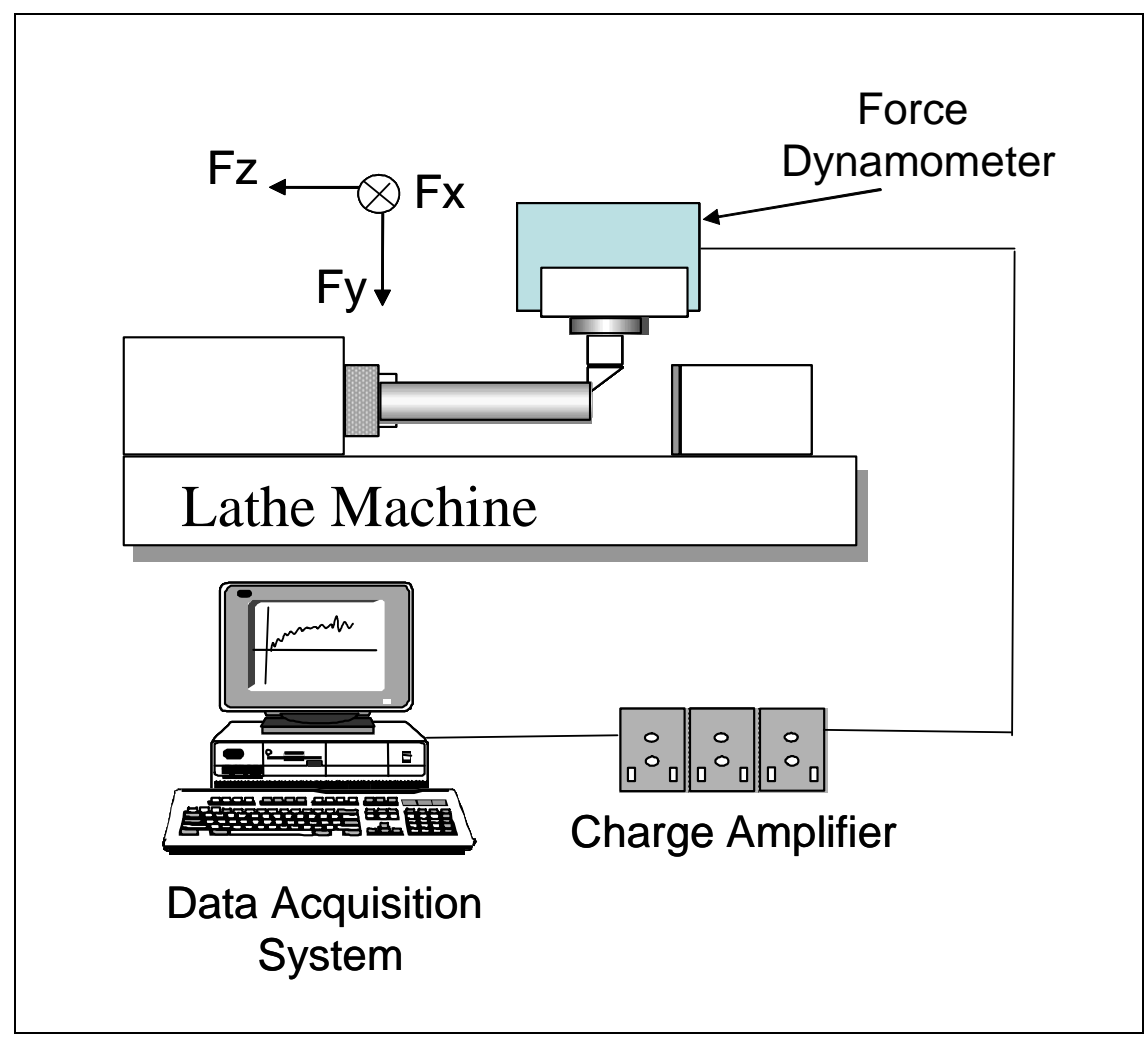

Figure 4: A schematic diagram of the experimental work. 


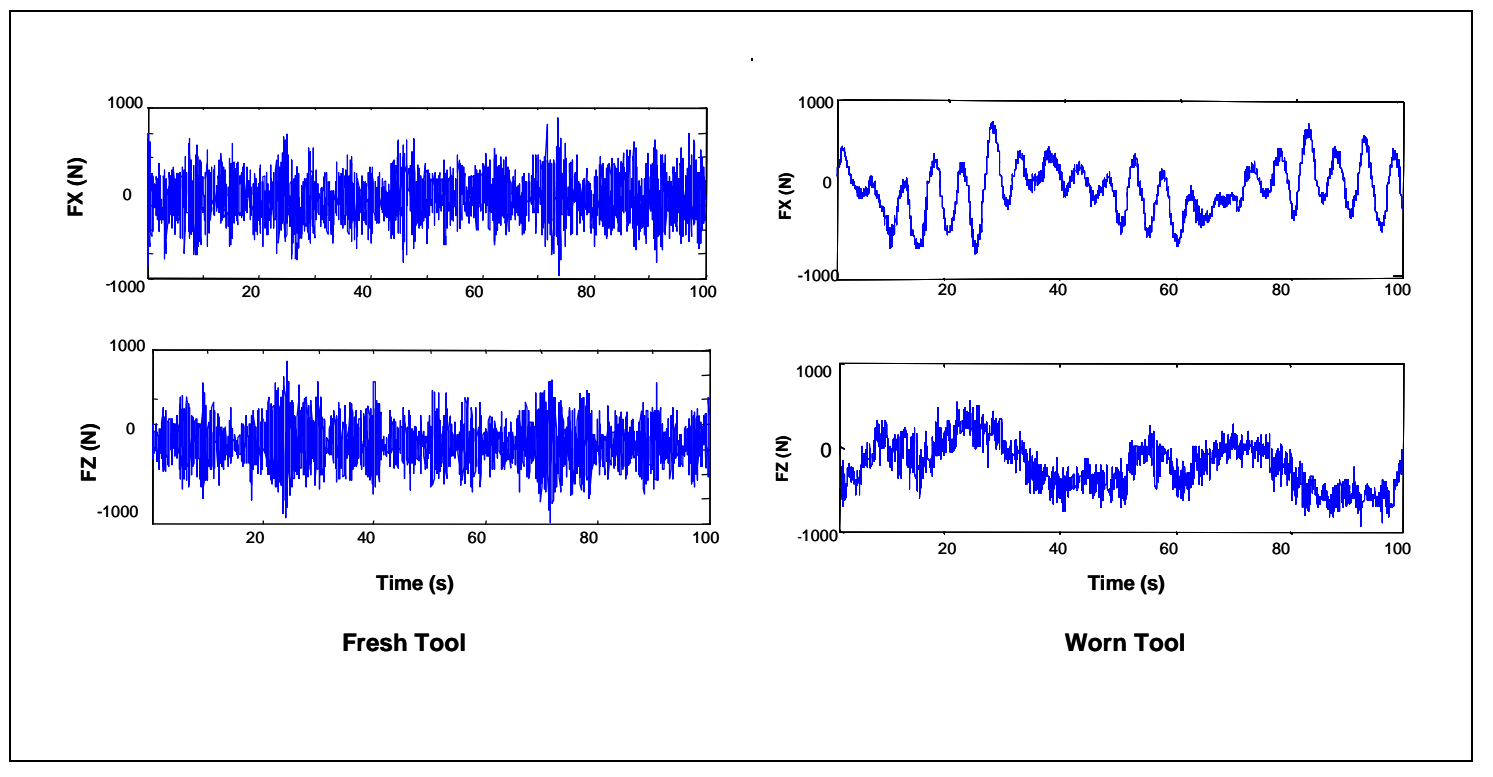

Figure 5: Example of the raw signals of the first tool. 

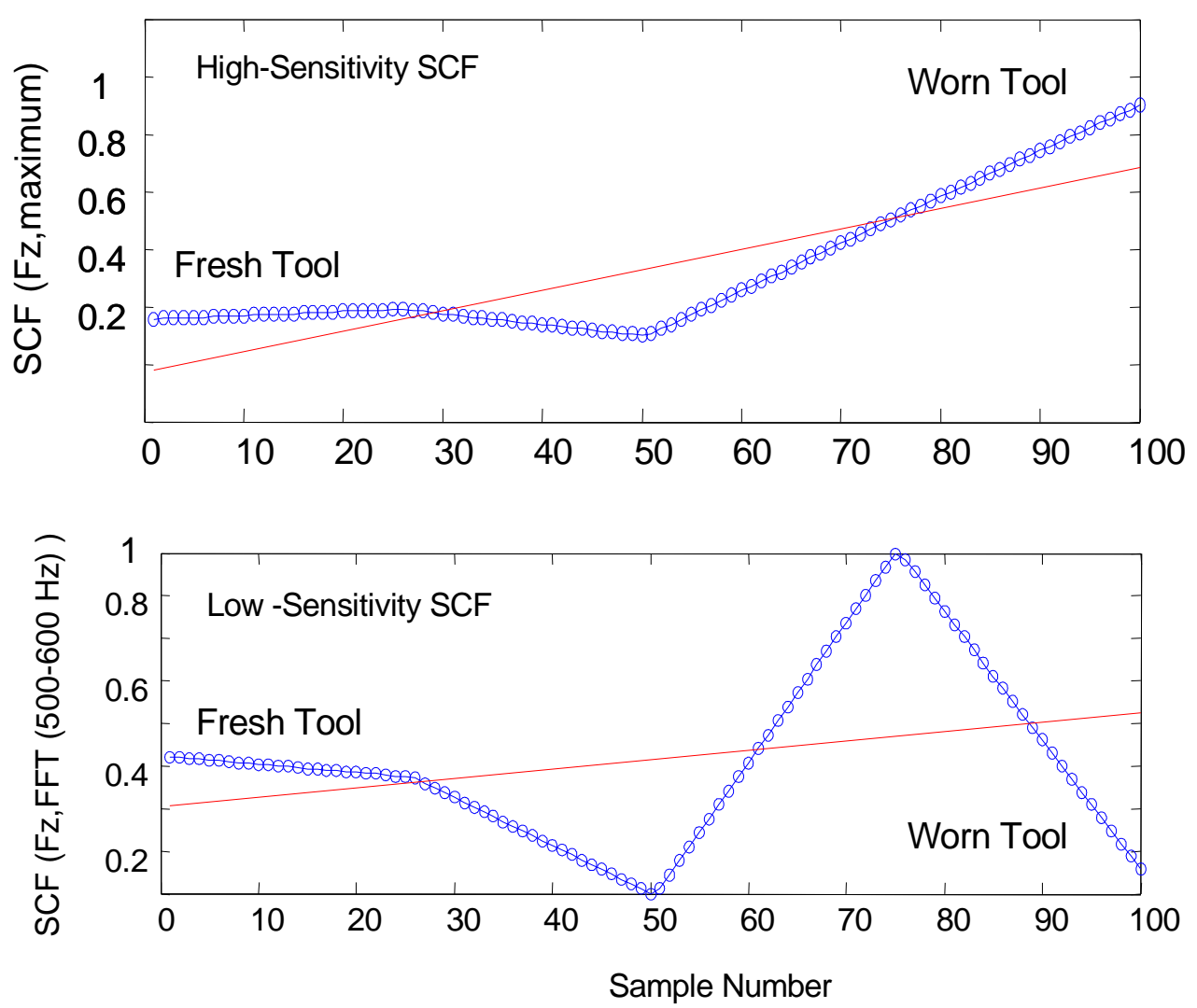

Figure 6: Example of low and high sensitivity SCF. 


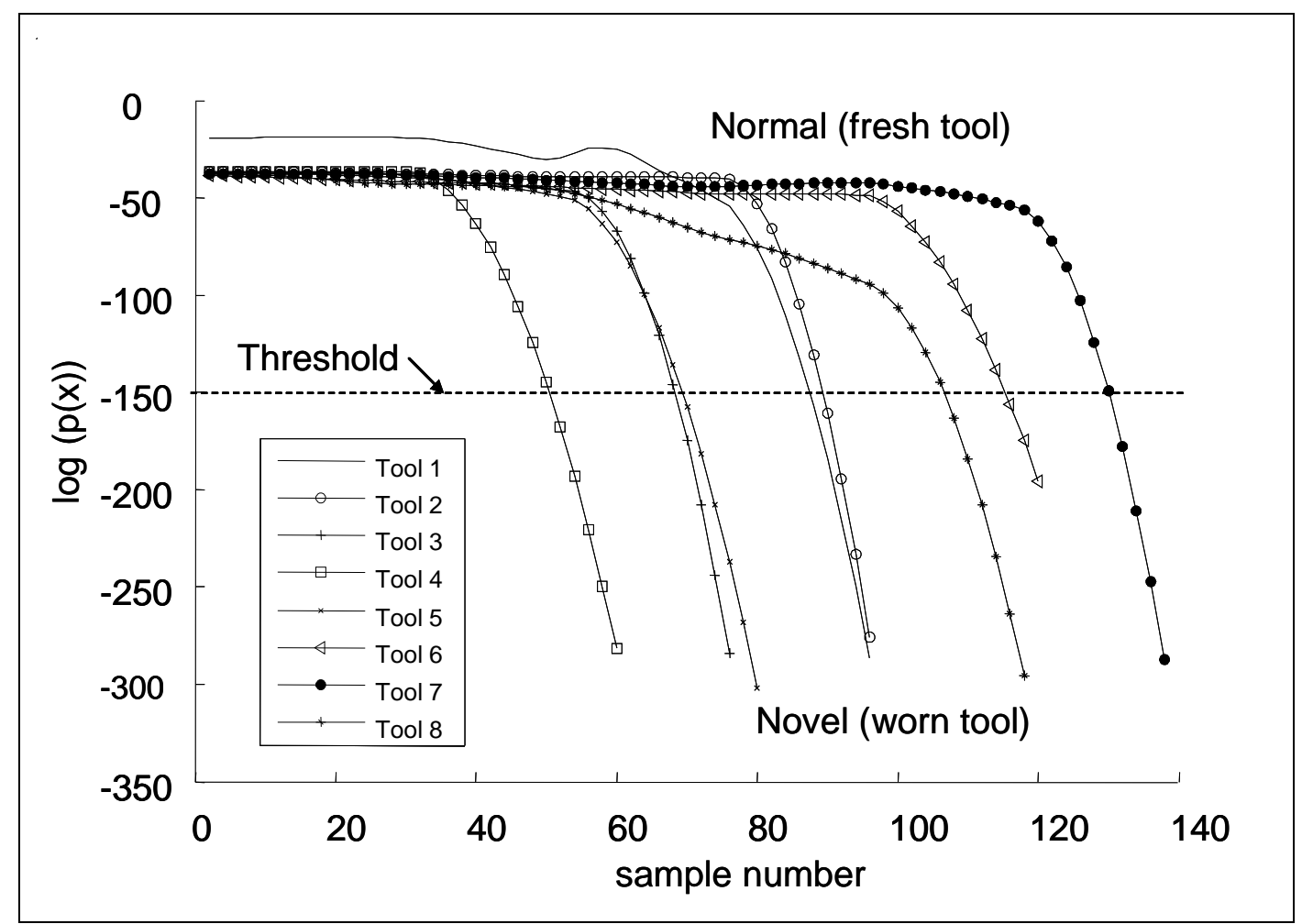

Figure 7: The results of the novelty detection to detect gradual tool wear in turning.

Al-Habaibeh, A. and Gindy, N. (2000) "A new approach for systematic design of condition monitoring systems for milling processes" Journal of Materials Processing Technology, 107, 243-251.

Al-Habaibeh, A. and Gindy, N. (2001) "Self-Learning Algorithm for Automated Design of Condition Monitoring Systems for Milling Operations" International Journal of Advanced Manufacturing Technology, 18, 448-459.

Al-Habaibeh, A. and Parkin, R. (2003) "An autonomous low-Cost infrared system for the on-line monitoring of manufacturing processes using novelty detection" International Journal of Advanced Manufacturing Technology, 22, 249-258.

Al-Habaibeh, A., Zorriassatine, F. and Gindy, N. (2002) "Comprehensive experimental evaluation of a systematic approach for cost effective and rapid design of condition monitoring systems using Taguchi method" Journal of Materials Processing Technology, 124, 372-383.

Al-Habaibeh, A., Zorriassatine, F., Parkin, R. and Jackson, M. (2005) "Application of Infrared Technology for quality Control of Diesel Engine Glowplug" Proc ImechE, part B, Journal of Engineering Manufacture, 219.

Azouzi, R. and Guillot, M. (1997) "On-line prediction of surface finish and dimensional deviation in turning using neural network based sensor fusion" International Journal of Machine Tools and Manufacture, 37, 1201-1217.

Barschdorff, D., Monostori, L., Kottenstede, T. and Warnecke, G. (1993) "Cutting Tool Monitoring in Turning Under Varying Cutting Conditions: An Artificial Neural Network Approach" Industrial and engineering applications of artificial intelligence and expert systems, 353-359.

Bishop, C. M. (1995) "Neural Networks for Pattern Recognition" Claredon Press. 
Cakir, M. C. and Isik, Y. (2005) "Detecting tool breakage in turning aisi 1050 steel using coated and uncoated cutting tools" Journal of Materials Processing Technology, 159, 191-198.

Choudhury, S. K. and Kishore, K. K. (2000) "Tool wear measurement in turning using force ratio" International Journal of Machine Tools and Manufacture, 40, 899-909.

Chung, E. S., Chiou, Y. S. and Liang, S. Y. (1993) "Tool wear and chatter detection in turning via time series modelling and frequency band averaging" ASME PED, 64, 351-358.

Das, S., Roy, R. and Chattopadhyay, A. B. (1996) "Evaluation of wear of turning carbide inserts using neural networks" International Journal of Machine Tools and Manufacture, 36, 789-797.

Dimla, J., D. E., Lister, P. M. and Leighton, N. J. (1997) "Neural network solutions to the tool condition monitoring problem in metal cutting--A critical review of methods" International Journal of Machine Tools and Manufacture, 37, 1219-1241.

Dimla Sr., D. E. (2000) "Sensor signals for tool-wear monitoring in metal cutting operations--a review of methods" International Journal of Machine Tools and Manufacture, 40, 1073-1098.

Dimla Sr., D. E. and Lister, P. M. (2000a) "On-line metal cutting tool condition monitoring.: I: force and vibration analyses" International Journal of Machine Tools and Manufacture, 40, 739-768.

Dimla Sr., D. E. and Lister, P. M. (2000b) "On-line metal cutting tool condition monitoring.: II: toolstate classification using multi-layer perceptron neural networks" International Journal of Machine Tools and Manufacture, 40, 769-781.

Dornfeld, D. (1990) "Neural network sensor fusion for tool condition monitoring" Annals CIRP, 39, 101105.

Dornfeld, D. A. and Lan, M. S. (1984) "In-process tool fracture detection." J. Engng Mater. Technol. 106, 111-118.

Elanayar, S., Shin, T. and Kumara, S. (1990) "Machining condition monitoring of automation using neural networks" Asme, 44, 85-100.

Elanyar, S. and Shin, Y. C. (1995) "Robust tool estimation with radial basis function neural networks" $J$. Dynamic Systems, Measurement and Control, 117, 459-467.

Etter, M. (1993) ""Engineering Problem Solving wih Matlab", Englewood Cliffs: Prentice Hall".

Fukunaga, K. (1990) "Introduction to Statistical Pattern Recognition", Academic Press, Boston; London.

Hong, G. S., Rahman, M. and Zhou, Q. (1996) "Using neural network for tool condition monitoring based on wavelet decomposition" International Journal of Machine Tools and Manufacture, 36, 551566.

Kakade, S. and et, a. (1995) "Monitoring of tool status using intelligent acoustic emission sensing and decision based neural network" Int. Conference on Industrial Automation \& Control, 25.

Ko, T. J. and Cho, D. W. (1994) "Cutting state monitoring in milling by a neural network" International Journal of Machine Tools and Manufacture, 34, 659-676.

Lee, J. H., Kim, D. E. and Lee, S. J. (1996) "Application of neural networks to flank wear prediction" Mechanical Systems and Signal Processing, 10, 265-276.

Lee, K. S., Lee, L. C. and Teo, S. C. (1992) "On- line tool wear monitoring using a PC." Journal of Material Process Technology, 29, 3-13.

Li, X. (2002) "A brief review: acoustic emission method for tool wear monitoring during turning" International Journal of Machine Tools and Manufacture, 42, 157-165.

Martin, K. F. (1994) "A review by discussion of condition monitoring and fault diagnosis in machine tools" International Journal of Machine Tools and Manufacture, 34, 527-551.

Masory, O. (1991) "Detectionof tool wear using multi-sensor readings defused by artificial neural network" SPIE, Int. Soc for optical Eng., 1469, 515-525.

Monostori, L. (1993) "A step towards intelligent manufacturing modelling and monitoring of manufacturing processes through artificial neural networks" Annals CIRP, 42, 485-488. 
Nabney, I. and Bishop, C. M. (2000) In Secondary "Netlab Neural Networks Software, (http://www.ncr.uk/netlab/index.html)"Neural Computing Research Group, Information Engineering, Aston University, Birmingham B4 7ET, UK, Oct 2000.

Parzen, E. (1962) "Stochastic Processes", Holden-Day.

Purushothaman, S. and Srinivasa, Y. G. (1994) "A back-propagationalgoruthm applied to tool wear monitoring" International Journal of Machine Tools and Manufacture, 34, 625-631.

Rahman, M., Zhou, Q. and Hong, G. S. (1995) "On-line cutting state recognition in turning using a neural network" International Journal of Advanced Manufacturing Technology, 10, 87-92.

Rangwala, S. and Dornfield, D. A. (1987) "Sensor Integration Using Neural Networks for Intelligent Tool Condition Monitoring" Trans. ASME J. Engmg Ind, 110.

Scheffer, C. and Heyns, P. S. (2004) "An industrial tool wear monitoring system for interrupted turning" Mechanical Systems and Signal Processing, 18, 1219-1242.

Sick, B. (2002) "On-Line and Indirect Tool Wear Monitoring in Turning with Artificial Neural Networks: A Review of More Than a Decade of Research." Mechanical Systems and Signal Processing, 16, 487-546.

Sokolowski, A., Kolka, A. and Kosomol, J. (1997) "A New Approach to Aid Designing of Monitoring Systems" Intrnational MATADOR.

Sokolowski, A. and Kosomol, J. (1996) "Intelligent Monitoring System Deigner, Japan/USA Symposium on Flexible Automation" ASME, 2.

Specht, D. F. (1990) "Probabilistic Neural Networks" Neural Networks, 3, 109-118.

Tanner, R. and Loh, N. K. (1994) "A taxonomy of multi-sensor fusion" Journal of Manufacturing Systems, 11, 314-325.

Tansel, I. N. and McLaughlin, C. (1993) "Detection of tool breakage in milling operations--I. The time series analysis approach" International Journal of Machine Tools and Manufacture, 33, 531-544.

Tlusty, J. and Andrews, G. C. (1983) " A critical review of sensors for unmanned machining" Ann. CIRP 32, 563-572.

Wang, Z. and Dornfeld, D. A. (1992) "In-Process Tool Wear Monitoring Using Neural Networks", Japan/USA Symposium on Flexible Automation." ASME, 1.

Yao, Y. and Fang, X. (1993) "Assessment of chip forming patterns with tool wear progression in machining via neural networks" international Journal of machine Tool and Manufacture, 33, 89102.

Zhou, Q., Hong, G. S. and Rahman, M. (1995) "A new tool life criterion for tool condition monitoring using a neural network" Engineering Applications of Artificial Intelligence, 8, 579-588.

Zorriassatine, F. (2000), "Application of Neural Networks for Detection of Special Causes in Multivariate Statistical Control", PhD Thesis, University of Nottingham, 